\title{
Transferência de modelos de previsão de acidentes entre cidades brasileiras
}

\author{
Flávio José Craveiro Cunto', Christine Tessele Nodari², Heloisa Maria Barbosa ${ }^{3}$
}

\begin{abstract}
Resumo: Os modelos de previsão de acidentes (MPA) são expressões que relacionam a frequência dos acidentes de trânsito com atributos geométricos e operacionais da via e lidam, com relativo sucesso, com o elevado grau de aleatoriedade desse fenômeno. O desenvolvimento desses modelos exige esforços consideráveis para a obtenção de dados em quantidade e qualidade adequados para a modelagem. A utilização de modelos desenvolvidos em outras jurisdições pode ser uma alternativa menos onerosa do que o desenvolvimento de modelos específicos para cada localidade. $O$ objetivo deste trabalho é avaliar a transferibilidade de MPA para interseções semaforizadas entre cidades brasileiras. Modelos previamente desenvolvidos para Fortaleza e Belo Horizonte foram calibrados e validados para a estimação dos acidentes na cidade de Porto Alegre. Os resultados indicaram que a transferência de MPA entre jurisdições é uma alternativa que deve ser usada com parcimônia, sendo desejável investir esforços no desenvolvimento de modelos específicos para cada jurisdição.
\end{abstract}

Palavras-chave: Modelos de Previsão de Acidentes. Transferência de MPA. HSM. Acidentes de trânsito.

\begin{abstract}
Accident prediction models (APM) are expressions that relate the frequency of traffic accidents with road geometric and operational attributes and deal with relative success, with the high degree of randomness related to this phenomenon. The development of these models requires considerable efforts to obtain data on quantity and quality suitable for modeling. The use of models developed in other jurisdictions may be a less costly alternative than the development of specific models for each location. The objective of this study is to evaluate the transferability of APM for signalized intersections between cities. Models previously developed for Fortaleza and Belo Horizonte were calibrated and validated for the estimation of accidents in the city of Porto Alegre. The results indicated that the use of APM between jurisdictions is an alternative that should be used with caution, and it is desirable to invest efforts in developing specific models for each jurisdiction.
\end{abstract}

Keywords: Accident Prediction Models. Transferability of APM. HSM. Traffic accidents.

\section{INTRODUÇÃO}

A preocupação com os índices de acidentalidade, tanto em centros urbanos como em rodovias rurais, tem motivado o desenvolvimento de diversos estudos na área de segurança viária. Dentre eles, destacam-se os estudos que tratam do desenvolvimento de modelos de previsão de acidentes (MPA). Tais modelos surgem como uma importante ferramenta para o diagnóstico das reais condições de segurança de diferentes configurações do ambiente viário.

Para o desenvolvimento dos MPA é necessária uma abrangente série histórica de dados confiáveis relativos à frequência de acidentes e às características geométricas e operacionais dos locais onde ocorreram tais acidentes. Esses dados, quando disponíveis, tendem a ser

\footnotetext{
1 Departamento de Engenharia de Transportes, Universidade Federal do Ceará (flaviocunto@det.ufc.br).

2 Laboratórios de Sistemas de Transportes - LASTRAN, Departamento de Engenharia de Produção e Transportes, Universidade Federal do Rio Grande do Sul (piti@producao.ufrgs.br).

3 Departamento de Engenharia de Transportes e Geotecnia, Universidade Federal de Minas Gerais (helobarb@etg.ufmg.br).
}

Manuscrito recebido em 15/05/2014 e aprovado para publicação em 22/09/2014. Este artigo é parte de TRANSPORTES v. 22, n. 3, 2014. ISSN: 2237-1346 (online).

DOI: http://dx.doi.org/10.14295/transportes.v22i3.790. coletados e armazenados por diferentes setores, o que compromete sua compatibilização. Alguns dos problemas recorrentes relativos à obtenção de dados necessários para o desenvolvimento de MPA referem-se à continuidade do período de coleta dos dados e confiabilidade dos registros.

Alternativamente ao desenvolvimento de MPA, é possível considerar a utilização de modelos já desenvolvidos em outras jurisdições desde que devidamente calibrados com dados locais. A calibração de MPA é menos exigente quanto à disponibilidade de dados tornando-se uma alternativa viável quando estes não estiverem disponíveis em quantidade e/ou qualidade desejada. O Highway Safety Manual - HSM (AASHTO, 2010) disponibiliza um procedimento de calibração e oferece modelos base, desenvolvidos para ambientes norteamericanos. No entanto, esses modelos refletem as características locais dos seus dados de origem, o que traz um nível de questionamento em relação à validade desses modelos para outras realidades, principalmente para o Brasil.

Algumas cidades brasileiras já despenderam esforços no desenvolvimento de MPA. Entre elas tem-se a cidade de Fortaleza (Cunto 
et al., 2012), Brasília (Claude, 2012) e Belo Horizonte (Costa e Barbosa, 2011). Esses esforços resultaram na proposição de modelos específicos para as características locais dessas cidades. Naturalmente, esses modelos tendem a apresentar melhores condições de serem calibrados para outras cidades brasileiras do que os propostos pelo HSM, desenvolvidos com base em dados norte-americanos.

Neste contexto, este artigo tem por objetivo avaliar a transferência de MPA entre cidades brasileiras. Para atender a este objetivo, modelos desenvolvidos em Fortaleza e Belo Horizonte foram calibrados e validados para a estimação dos acidentes de trânsito na cidade de Porto Alegre.

O presente artigo está estruturado em cinco seções incluindo esta introdução. Na seção 2 são apresentados conceitos e experiências relativas à transferência de MPA; a seção 3 apresenta o método de trabalho adotado; na seção 4 os resultados obtidos e, por fim, a seção 5 contém as conclusões do estudo.

\section{TRANSFERÊNCIA DE MPA ENTRE JURISDIÇÕES}

O interesse na verificação da aplicabilidade de MPA desenvolvidos em jurisdições diferentes advém basicamente da necessidade de confirmação das diferenças no ambiente geral da segurança viária entre as duas jurisdições e também, da possibilidade do desenvolvimento de modelos que possam ser utilizados de forma mais generalizada por uma região ou mesmo país.

Vogt e Bared (1998) analisaram as diferenças entre MPA desenvolvidos para segmentos viários e interseções semaforizadas dos estados de Minnesota e Washington. Para os segmentos viários foram desenvolvidos modelos comuns nos quais o estado era representado por uma variável binária a qual apresentou uma diferença média de quase $15 \%$ nos acidentes de trânsito estimados. Para o caso das interseções, foram desenvolvidos MPA para os dados de Minnesota, os quais foram utilizados para estimar os acidentes das entidades de Washington. A comparação dos valores estimados com os observados em Washington gerou um fator multiplicador que, de acordo com os autores, permite a utilização dos modelos base desenvolvidos para Minnesota em Washington.
De forma mais específica, observa-se recentemente um esforço considerável na avaliação da transferência dos modelos contidos no HSM, considerando-se que esse manual é destinado a fornecer orientação para os profissionais de segurança viária tornando-se um padrão de prática internacional. Diferenças nos sistemas de informação de acidentes, legislação local, comportamento do motorista e questões relativas ao ambiente entre jurisdições podem, entretanto, prejudicar o uso universal do método de previsão do HSM (Sun et al., 2006, Xie et al., 2011, Sacchi et al., 2012).

O método de previsão de acidentes do HSM apresenta três componentes principais, o MPA para condições básicas, os Fatores de Modificação de Acidentes (CMF - crash modification factors) e o fator de calibração para ajustar o MPA às condições locais. A fórmula pode ser representada por (AASHTO, 2010):

$N_{\text {previsto, } i}=N_{M P A, i} \times\left(C M F_{1, i} \times C M F_{2, i}\right.$ $\left.\times \ldots \times C M F_{y, i}\right) \times C_{x}$

em que:

Nprevisto, $i$ : número de acidentes previstos para um ano específico em um local

$N_{M P A, i}:$ número de acidentes previstos determinado para a condição básica do MPA desenvolvido para o local $i$

$C M F y, i$ : fator de modificação de acidentes do MPA para o local $i$

$C x$ : fator de calibração para ajustar o MPA para as condições locais.

Os MPA básicos no HSM normalmente são calibrados pressupondo a distribuição binomial negativa para o número de acidentes observados, que é (na maioria dos casos) mais adequada para modelar a alta variabilidade natural de dados de acidentes. Os CMF são utilizados para modificar a frequência de acidentes previstos a partir do MPA base, considerando atributos específicos das entidades, como faixas de conversão à esquerda e à direita, condições de iluminação, entre outros. No procedimento de calibração recomendado no HSM (AASHTO, 2010), Cx, é obtido como se segue:

$$
C_{x}=\frac{\sum_{\text {todososlocais }} \text { acidentes observados }}{\sum_{\text {todososlocais }} \text { acidentes previstos }}
$$


em que a soma dos acidentes previstos é calculada a partir da Equação 1 sem o $C x$.

Os resultados da modelagem $\left(N_{\text {previsto, } i}\right)$ podem então ser empregados para melhorar a estimativa do número esperado de acidentes no local através do método empírico de Bayes (EB) de acordo com:

$N_{\text {esperado }, i}=w_{i} \times N_{\text {previsto }, i}+\left(1-w_{i}\right) \times N_{\text {observado, } i}$

$$
w_{i}=\frac{1}{1+k\left(\sum_{\text {todos_anos_de_estudo }} N_{\text {previsto }}\right)}
$$

em que:

$N_{\text {esperado, } i: \text { número de acidentes espera- }}$ dos para o período de estudo para o local $i$

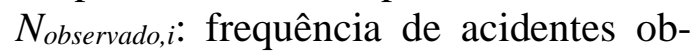
servados no local $i$ no período de estudo

$w_{i}$ : ajuste ponderado para ser usado na estimativa do modelo de previsão - local $i$

$k$ : parâmetro de dispersão da distribuição binomial negativa.

O método EB é considerado crucial para compensar o fenômeno de regressão à média (FRM) normalmente existente em estudos de segurança viária. Dentre os problemas mais comuns atribuídos ao FRM estão a escolha errônea de pontos críticos e a superestimação do efeito de intervenções no desempenho da segurança viária (Hauer, 1997; HSM, 2010).

A grande maioria dos estudos relacionados ao uso do HSM em diferentes jurisdições tem se concentrado em estimar o coeficiente $C_{x}$ para diferentes tipos de entidades (Sun et al., 2006, Xie et al., 2011, Sacchi et al., 2012). Os resultados indicam considerável discrepância entre os coeficientes seja em comparações dentre ou fora dos EUA.

Sawalha e Sayed (2006) questionam o processo de calibração jurisdicional dos modelos sem uma avaliação mais detalhada da compatibilidade entre a dispersão observada nos dados do modelo original e os dados da amostra a ser calibrada. Para esses autores, o parâmetro de forma $k$ (também chamado de parâmetro de dispersão $\phi$ ) da distribuição binomial negativa também deve ser recalibrado de forma a representar de forma mais precisa a dispersão dos dados da nova amostra. Neste caso a nova estimativa do parâmetro de dispersão da distri- buição binomial negativa por ser expresso por:

$$
k=\frac{\frac{1}{N} \sum_{i=1}^{N} \mu_{i}^{2}}{\frac{1}{N} \sum_{i=1}^{N}\left[\left(y_{i}-\mu_{i}\right)^{2}-\mu_{i}\right]}
$$

em que:

$y_{i}$ : contagem de acidentes observados no local $i$ ( $N$ é o número de locais)

$\mu_{i}$ : número esperado de acidentes no local $i$ (equivalente ao $N_{\text {previsto }, i}$ )

Vogt e Bared (1998) afirmam que se (1) a distribuição binomial negativa associada ao novo conjunto de dados é compatível, ou seja, as previsões do modelo estimam as médias das observações de acidentes no novo conjunto de dados, e (2) as observações no novo conjunto de dados são independentes, então as equações $5 \mathrm{a}$ e $5 \mathrm{~b}$ fornecem o valor esperado e o desvio padrão do $\chi^{2}$ de Pearson:

$$
\begin{aligned}
& E\left(\chi_{p}^{2}\right)=N \\
& \sigma\left(\chi_{p}^{2}\right)=\sqrt{2 N\left(1+\frac{3}{k}\right)+\sum_{i=1}^{N} \frac{1}{\mu_{i}\left(1+\frac{\mu_{i}}{k}\right)}}
\end{aligned}
$$

em que $N$ : número de observações no novo conjunto de dados.

A relação seguinte, chamada de escore $z$, mede quanto o $\chi^{2}$ estimado dista de seu valor esperado:

$$
z=\frac{\chi_{p}^{2}-E\left(\chi_{p}^{2}\right)}{\sigma\left(\chi_{p}^{2}\right)}
$$

Sawalha e Sayed (2006) utilizaram então o escore $z$ para avaliar ambas as alternativas (com e sem a recalibração de k) em relação à adequação dos dados observados ao modelo original. Valores de $z$ perto de zero validam o modelo de transferência. Contudo, Vogt e Bared (1998) não indicam os limites de distância do zero além do qual o escore $z$ deixa de validar o modelo de transferência. Isto pode ser devido ao fato da distribuição matemática de $\mathrm{z}$ ser desconhecida, o que não permite estabele- 
cer a zona de aceitação e rejeição para a hipótese de um determinado modelo estar correto para um conjunto de dados (Sawalha e Sayed, 2006).

Além dos testes mencionados, Sacchi et al.(2012) exploraram a aplicação de gráficos do número observado e previsto de acidentes de trânsito, gráficos de resíduos acumulados e índices como o desvio médio absoluto (MAD) na avaliação do desempenho dos modelos do HSM recalibrados para a cidade de Turim, Itália. Os resultados indicaram que, para a jurisdição italiana, recomendam-se esforços no senti- do do desenvolvimento de seus próprios modelos de previsão de acidentes.

\section{PROCEDIMENTO METODOLÓGICO}

A avaliação da transferência dos MPA desenvolvidos em Fortaleza e Belo Horizonte para o ambiente urbano de Porto Alegre foi estruturado em quatro etapas conforme ilustra o fluxograma da Figura 1. A seguir serão detalhadas as atividades que compõem cada uma das etapas metodológicas.
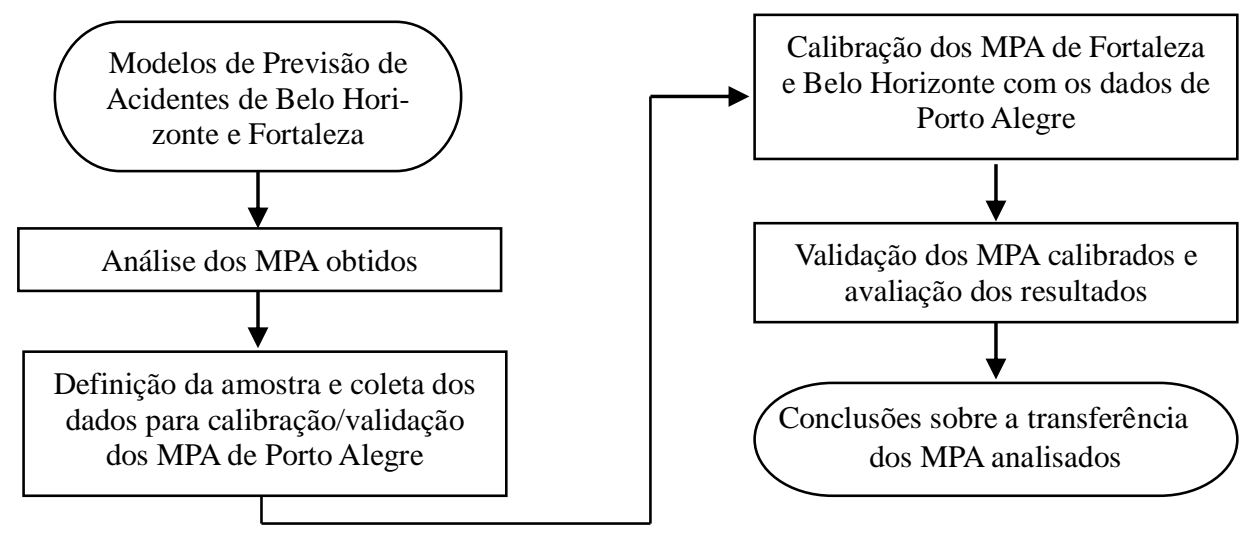

Figura 1 - Fluxograma do método de calibração/validação proposto

\subsection{Análise dos MPA de Fortaleza e Belo Horizonte}

Nessa etapa foi feita a análise dos MPA de Fortaleza e Belo Horizonte para a identificação do tipo de entidade considerada e das variáveis adotadas nos modelos de forma a orientar o processo de amostragem das entidades de Porto Alegre. Os MPA considerados neste estudo referem-se a interseções de vias arteriais urbanas. Tanto o modelo de Belo Horizonte quanto o de Fortaleza foram desenvolvidos para interseções de quatro aproximações.

$\mathrm{O}$ procedimento de modelagem adotado nas duas cidades seguiu a formulação geral comumente adotada para a calibração dos MPA apresentada em Cunto et al. (2012) e Costa e Barbosa (2011). Os modelos calibrados de melhor desempenho utilizaram as mesmas variáveis explicativas, o volume diário médio anual (VDMA) e o número total de faixas da interseção.

Ambos os modelos foram desenvolvidos para o ano de 2009 utilizando uma amostra com 101 interseções em Fortaleza e 220 interseções para o caso de Belo Horizonte. Foram consideradas as interseções de quatro aproximações por se constituírem no tipo de entidade mais frequente nos bancos de dados de acidentes das cidades pesquisadas. Ressalta-se que para o caso de Fortaleza foram utilizados todos os acidentes de trânsito como variável resposta e no caso de Belo Horizonte, consideraram-se somente os acidentes com vítimas feridas e fatais. Os modelos desenvolvidos correspondem às seguintes expressões:

Fortaleza:

$$
Y_{F_{i}}=0,0051 \times\left(v d m a^{0,52}\right) \times e^{0,28 n f x}
$$

Belo Horizonte:

$$
Y_{B H_{i}}=0,0064 \times\left(v d m a^{0,51}\right) \times e^{0,14 n f x}
$$

em que:

$Y_{F_{i}}$ : número total esperado de acidentes por ano na interseção $i$

$Y_{B H_{i}}$ : número esperado de acidentes com 
vítimas feridas e fatais por ano na interseção $i$ $v d m a$ : volume diário médio total da interseção ção.

$$
n f x \text { : número total de faixas da interse- }
$$

Os parâmetros de dispersão $(k)$ da distribuição binomial negativa foram estimados em 4,47 e 10,25 para os dados de Fortaleza e Belo Horizonte, respectivamente. Maiores detalhes sobre os modelos, como a estatística descritiva das variáveis testadas, valores do nível de significância das variáveis e avaliações de desempenho dos modelos podem ser encontrados em Barbosa et al. (2013).

\subsection{Definição da amostra e coleta dos dados para calibração/validação dos MPA de Porto Alegre}

Esta etapa consistiu no processo de amostragem das interseções semaforizadas de Porto Alegre e posterior coleta e tratamento dos dados necessários para o processo de calibração/validação. As informações sobre a frequência observada de acidente referem-se a um período de três anos, de 2008 a 2010 e foram obtidos junto à Empresa Pública de Transportes e Circulação (EPTC) de Porto Alegre. Neste estudo foram considerados todos os acidentes observados, ou seja, acidentes com danos materiais, vítimas feridas e vítimas fatais.

O VDMA utilizado foi estimado a partir de contagens no horário de pico e contagens estendidas realizadas nas interseções em um dia da semana (terça, quarta ou quinta) dentro do período de 2008 a 2010. Os procedimentos para expansão das contagens, bem como para corrigir a variação relacionada ao crescimento da frota, foram realizados através do cálculo de fatores de crescimento anuais, detalhados em Holz et al. (2012). No total, foram selecionados os dados de 55 interseções semaforizadas de quatro ramos.

Para compor o banco de dados de calibração e de validação, as observações foram consideradas sem distinção de ano. Dessa forma, cada interseção contribuiu para o banco de dados com três observações, uma para cada ano de análise. Assim, no total tem-se 165 observações (55 interseções em três anos diferentes). Esse procedimento viabiliza o uso de amostras com um maior número de observações, o que é desejável para a modelagem e para a calibração e validação dos MPA. Ressalta-se que possíveis correlações temporais entre as observações de uma mesma entidade surgidas com o procedimento proposto não deverão influenciar significativamente os resultados em virtude da pequena abrangência temporal da amostra (três anos).

As 165 observações foram divididas em dois grupos escolhidos de forma aleatória. O primeiro grupo, de 83 observações, foi destinado à calibração do fator $\mathrm{C}_{\mathrm{x}}$ (equação 1 ). $\mathrm{O}$ segundo grupo, composto por 82 observações, foi usado como amostra de validação. A Tabela 1 apresenta um resumo das variáveis utilizadas no processo de calibração e validação.

Tabela 1 - Estatística descritiva - variáveis de Porto Alegre

\begin{tabular}{ccccccccc}
\hline \multirow{2}{*}{ Variável } & \multicolumn{3}{c}{ Amostra de Calibração } & \multicolumn{4}{c}{ Amostra de Validação } \\
& Média & Sd. & Mín. & Máx. & Média & Sd. & Mín. & Máx. \\
\hline \multirow{2}{*}{ AADT } & 54.697 & 26.499 & 11.206 & 120.690 & 59.211 & 26.414 & 11.775 & 115.990 \\
$n f x$ & 6,9 & 2,0 & 4 & 11 & 7,3 & 2,1 & 4 & 11 \\
$Y$ & 10,1 & 9,0 & 0 & 41 & 12,3 & 10,5 & 0 & 41 \\
\hline
\end{tabular}

\subsection{Calibração dos modelos}

A etapa de calibração dos modelos consistiu inicialmente em estimar o valor do fator $\mathrm{C}_{\mathrm{x}}$ para os modelos de Fortaleza e Belo Horizonte a partir da razão entre o somatório da frequência de acidentes observados em Porto Alegre e o somatório das frequências estimadas diretamente com os MPA desenvolvidos pelas cidades do estudo (equação 1). Esse fator tem por finalidade ajustar possíveis diferenças entre as jurisdições envolvidas no processo de coleta, tabulação e armazenamento das informações sobre acidentes de trânsito e outros aspectos relativos ao ambiente de circulação como estado de conservação das vias, uso do solo, comportamento de condução dos motoristas, dentre outros. $\mathrm{O} \mathrm{C}_{\mathrm{x}}$ obtido para os modelos de Fortaleza e Belo Horizonte foram de 0,65 e 2,06 respectivamente.

A seguir verificou-se a adequação do parâmetro de dispersão $k$ da distribuição binomial 
negativa original para representar a dispersão dos dados de Porto Alegre. Os valores de $k$ foram recalibrados para os dois modelos a partir da equação 4 e os valores do escore $z$ (equação 6) foram estimados para as situações com e sem a recalibração de $k$. O uso dos valores recalibrados de $k$ somente é justificado nas situações em que o valor do escore $z$ se aproximou de zero após a calibração.

Finalmente o desempenho global dos modelos calibrados foi avaliado graficamente através da comparação entre os valores previstos e observados dos acidentes com a utilização dos gráficos de resíduos acumulados (cure plots). O gráfico de resíduos acumulados permite um exame visual da distribuição de resíduos do modelo ao longo da faixa dos valores das variáveis preditoras. Utilizou-se ainda o desvio médio absoluto (MAD) para comparar a precisão dos dois modelos calibrados. O MAD foi estimado por:

$$
M A D=\sum_{i=1}^{n}\left|\frac{\hat{Y}_{i}-Y_{i}}{n}\right|
$$

em que: terseção $i$

$Y_{i}$ : número previsto de acidentes na in-

Tabela 2 - Parâmetros estimados

\begin{tabular}{lllllllll}
\hline \multirow{2}{*}{ Parâmetro } & \multicolumn{7}{l}{ FORTALEZA } & \multicolumn{7}{c}{ BELO HORIZONTE } \\
\cline { 2 - 8 } & \multicolumn{2}{l}{ Calibração } & \multicolumn{2}{l}{ Validação } & \multicolumn{2}{c}{ Calibração } & \multicolumn{2}{c}{ Validação } \\
\hline & original & recalibrado & original & recalibrado & original & recalibrado & original & recalibrado \\
\hline$K$ & 4,47 & 5,76 & 4,47 & 5,76 & 10,25 & 5,10 & 10,25 & 5,10 \\
Escore $z$ & 3,40 & 5,00 & 7,59 & 10,41 & 3,15 & 0,62 & 8,37 & 4,11 \\
MAD & 4,33 & & 6,31 & & 4,26 & & 6,95 & \\
C & 0,65 & & & & 2,06 & & \\
\hline
\end{tabular}

O coeficiente $\mathrm{C}_{x}$ de 0,65 , obtido com o MPA de Fortaleza, aplicado aos dados de Porto Alegre pode ser interpretado de forma objetiva como, mantidas as mesmas condições de fluxo e número de faixas, o ambiente de Porto Alegre apresenta em média 35\% menos acidentes em comparação à Fortaleza. Uma análise superficial poderia sugerir que o trânsito de Fortaleza seria mais suscetível à ocorrência de acidentes do que o de Porto Alegre. Entretanto, fatores como diferenças no processo de coleta, tabulação, consolidação e armazenamento dos dados

\author{
$Y_{i}$ : número observado de acidentes na in- \\ terseção $i$ \\ $n$ : número total de interseções.
}

\subsection{Validação dos modelos calibrados}

A etapa de validação foi realizada com o objetivo de avaliar o comportamento dos modelos calibrados para a jurisdição de Porto Alegre em uma nova amostra de suas interseções semaforizadas. Espera-se que o modelo mais adequado apresente desempenho semelhante ao observado na amostra utilizada para calibração.

A avaliação de desempenho dos modelos nesta etapa foi obtida de forma similar à avaliação no procedimento de calibração. Foram usados os gráficos de frequências de acidentes observadas e previstas e o gráfico de resíduos. Além disso, foram usadas as métricas escore $z$ e MAD para confirmar as hipóteses em relação ao parâmetro de dispersão $(k)$ da distribuição binomial negativa e sobre o erro médio absoluto da amostra de validação.

\section{ANÁLISE DOS RESULTADOS}

Os parâmetros de dispersão, escore $z$, desvio médio absoluto, além dos coeficientes de calibração $C_{x}$ para os dados de calibração e validação das duas jurisdições estão apresentados na Tabela 2. de acidentes de trânsito, estimação das informações de fluxo veicular, bem como diferenças inerentes ao ambiente viário urbano de cada cidade (uso do solo, permissões de estacionamento, paradas de ônibus, entradas/saídas de lotes, etc.) limitam a associação direta do coeficiente $C_{x}$ a um trânsito menos ou mais seguro entre as localidades.

A calibração Belo Horizonte/Porto Alegre produziu um coeficiente $C_{x}$ de 2,06. Trata-se de um resultado até certo ponto esperado, uma vez que o MPA de Belo Horizonte estima somente 
o número esperado de acidentes com vítimas feridas e fatais. Como em Porto Alegre a variável resposta é o número total de acidentes de trânsito espera-se um fator de calibração que consiga expandir a abrangência do modelo de Belo Horizonte. Ressalta-se que a interpretação do $\mathrm{C}_{\mathrm{x}}$ nesse caso está sujeita ao mesmo conjunto de interações entre os fatores ambientais citados para o caso de Fortaleza.

Em relação ao comportamento do parâmetro $k$ observaram-se valores relativamente elevados para a amostra de calibração de Fortaleza e Belo Horizonte, sugerindo que os dados de Porto Alegre podem apresentar padrão de dispersão distinto nos dois casos. Entretanto, a recalibração de $k$ para o caso de Fortaleza aumentou o valor do escore $z$ indicando que neste caso, deve-se permanecer com o parâmetro original. Os valores para os dados de Belo Horizonte indicaram tendência contrária, ou seja, boa redução no escore $z$ corroborando a neces-

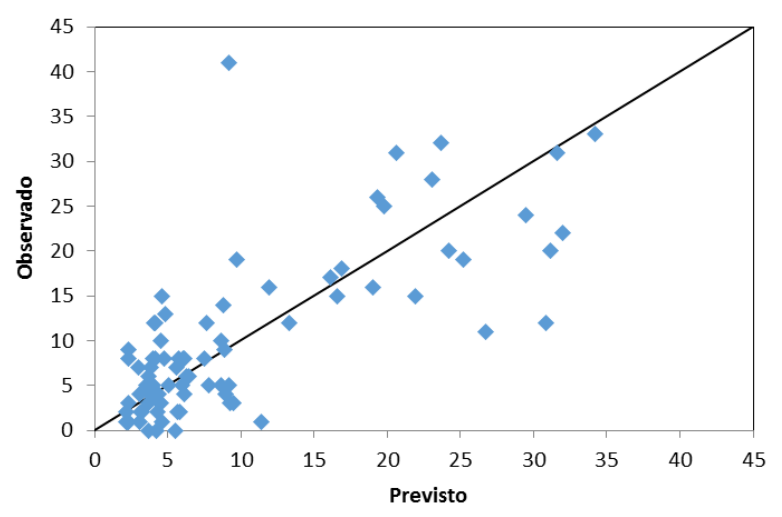

(a) sidade de recalibração do parâmetro $k$.

Os valores do desvio médio absoluto (MAD) foram de 4,33 e 4,26 acidentes para os modelos calibrados de Fortaleza e Belo Horizonte respectivamente. Esses resultados não possibilitam a atribuição de um relativo desempenho superior entre os modelos.

A análise visual entre os valores observados e previstos após a calibração (Figuras 2a e 2b) deixa aparente que o modelo calibrado de Belo Horizonte de maneira geral subestima os valores dos acidentes na região de poucos acidentes (até aproximadamente 12 acidentes) passando a superestimar depois disso (Figura 2b). Esse aspecto foi menos perceptível no modelo calibrado de Fortaleza (Figura 2a). Entretanto, observa-se que o modelo de Fortaleza apresenta-se mais disperso (em relação à reta de 45 graus) principalmente a partir de 12 acidentes em comparação ao modelo de Belo Horizonte.

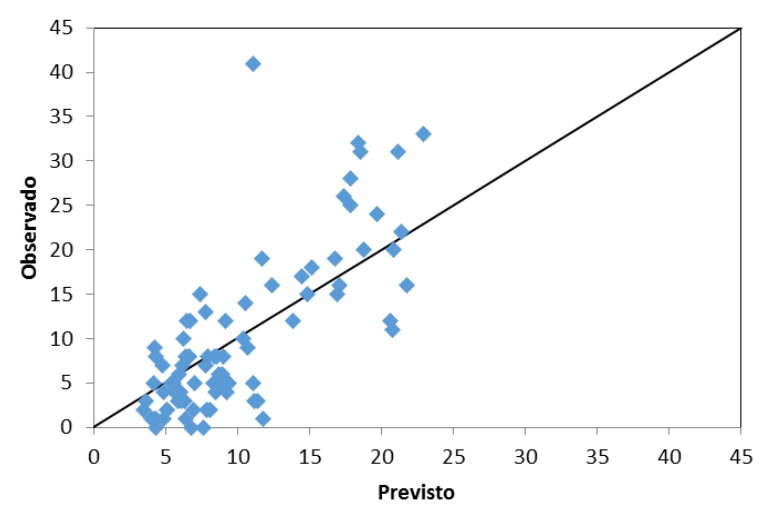

(b)

Figura 2 - Gráficos de frequência observada $x$ frequência prevista pelos modelos de Fortaleza (a) e Belo Horizonte (b) calibrados para Porto Alegre

Os gráficos de resíduos acumulados (Figuras $3 \mathrm{a}$ e $3 \mathrm{~b}$ ) sugerem que o modelo calibrado de Fortaleza se apresentou dentro dos limites de $\pm 2 \sigma_{d}$ em quase sua totalidade, enquanto que o modelo calibrado de Belo Horizonte ultrapassou este mesmo limite em uma faixa considerável de fluxo (de 30.000 a 80.000 veículos por dia). Além disso, a tendência decrescente no cure plot para o modelo de Belo Horizonte (Figura 3b) até 70.000 vpd e crescente a partir daí, indica tendência respectiva de super e subestimação dos acidentes de trânsito dessa feita em função do fluxo. É importante salientar que a aparente superioridade do modelo de Fortaleza pode estar relacionada ao fato de que os coeficientes de calibração das variáveis VDMA e número de faixas podem ser consideravelmente diferentes para representar todos os acidentes ao invés de somente os acidentes com vítimas feridas e fatais.

A avaliação com os gráficos de acidentes observados versus acidentes previstos (Figuras 4a e 4b) bem como a dos gráficos de dispersão (Figuras 5a e 5b) mostraram padrão consistente com os modelos desenvolvidos com a amostra de calibração, confirmando assim certa estabilidade dos coeficientes de calibração estabelecidos. 


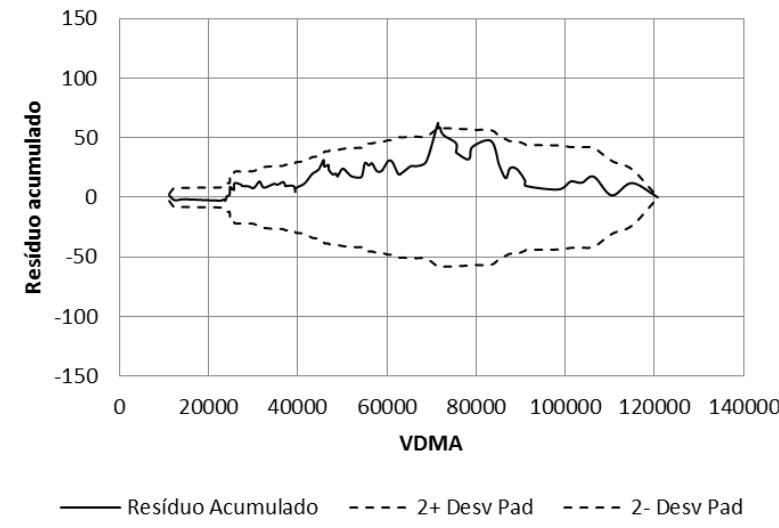

(a)

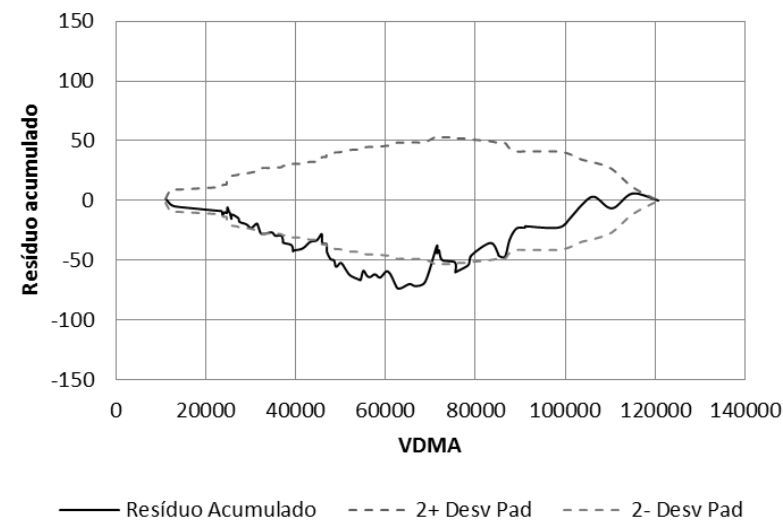

(b)

Figura 3 - Gráficos de resíduos acumulados dos modelos de Fortaleza (a) e Belo Horizonte (b) calibrados para Porto Alegre

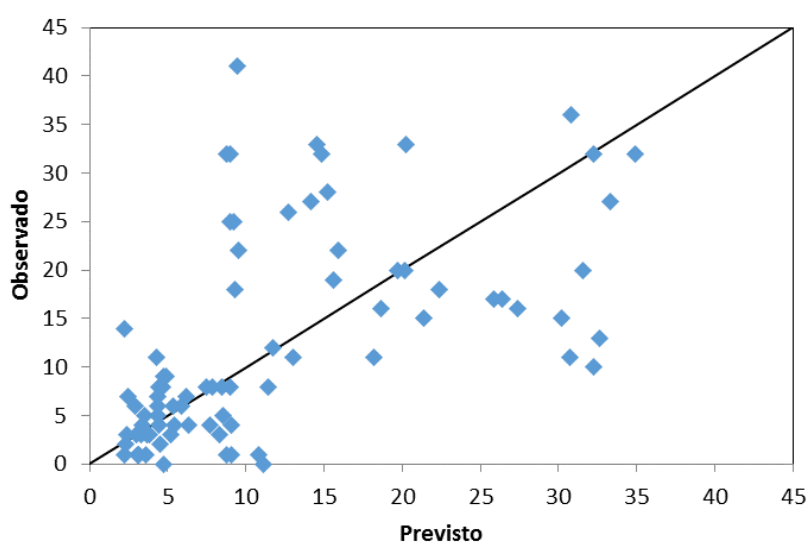

(a)

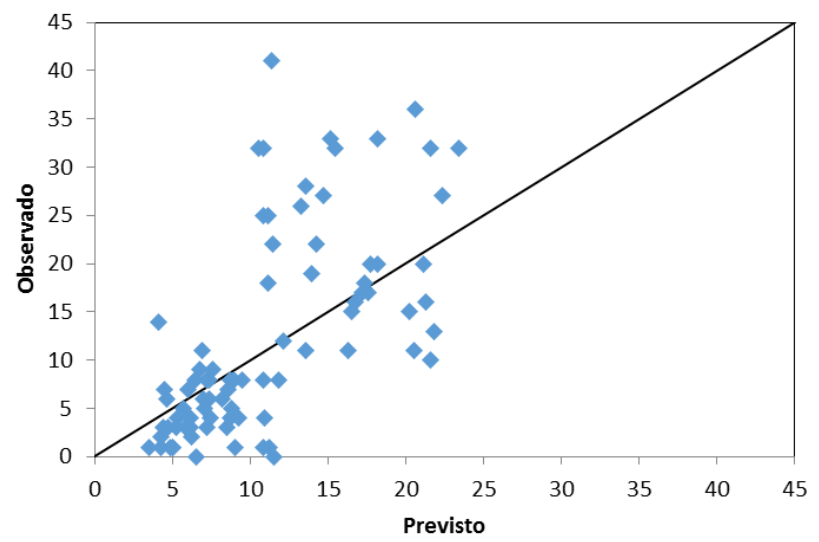

(b)

Figura 4 - Gráficos de frequência observada $x$ frequência estimada pelos modelos de Fortaleza (a) e Belo Horizonte (b) validados para Porto Alegre

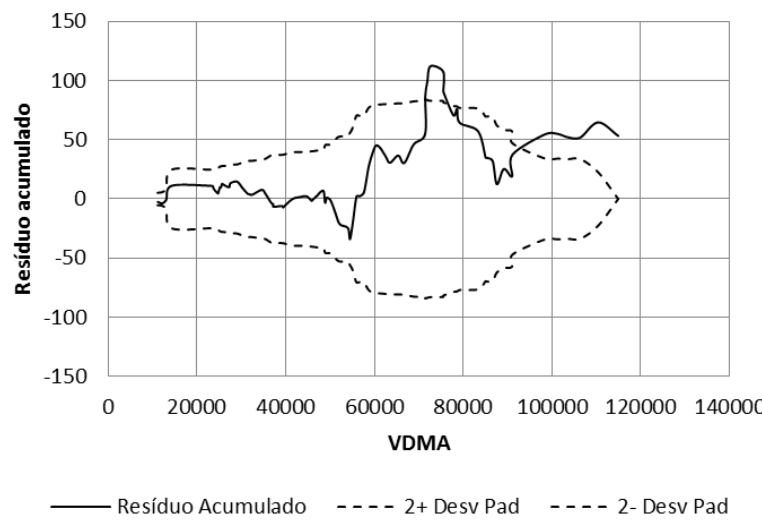

(a)

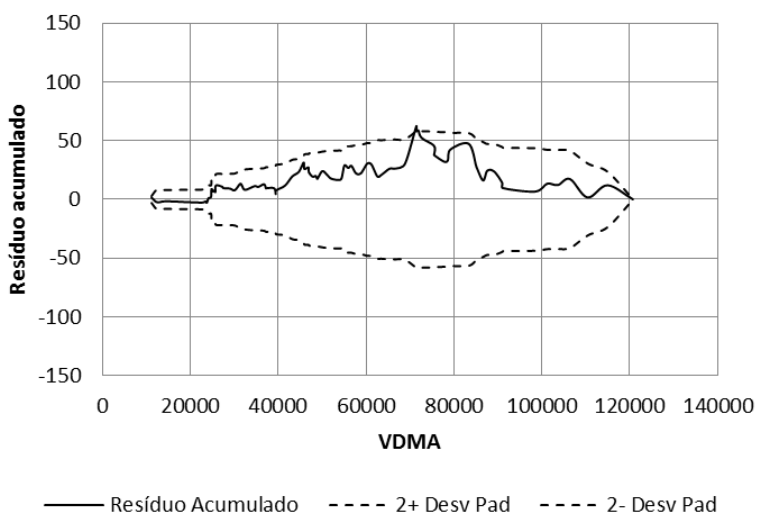

(b)

Figura 5 - Gráficos de resíduos acumulados dos modelos de Fortaleza (a) e Belo Horizonte (b) validados para Porto Alegre

\section{CONCLUSÕES}

A crescente demanda na qualificação das análises de segurança viária aponta para a necessidade da utilização de Modelos de Previsão de Acidentes (MPA). No entanto, o desenvolvimento de tais modelos exige esforços consideráveis para a obtenção de dados em quantidade e qualidade adequados para a modelagem. A transferência de modelos desenvolvidos em outras jurisdições pode ser uma alternativa me- nos onerosa do que o desenvolvimento de modelos específicos para cada localidade. Porém, o desempenho de modelos calibrados deve ser devidamente avaliado. É neste cenário que o presente artigo buscou analisar a transferibilidade de modelos entre cidades brasileiras.

Modelos desenvolvidos para interseções semaforizadas das cidades de Fortaleza e de Belo Horizonte foram calibrados e, posterior- 
mente, validados para um conjunto de dados de Porto Alegre. A amostra de calibração foi composta por 83 observações e a de validação por 82 observações. Os dados se referem a um período de três anos (2008 a 2010) e, em virtude da pequena abrangência temporal foram desconsideradas possíveis correlações temporais entre as observações de uma mesma entidade.

Os coeficientes de calibração encontrados foram $C_{x}=0,65$ para $o$ MPA de Fortaleza e $\mathrm{C}_{\mathrm{x}}=2,06$ para o MPA de Belo Horizonte. É importante destacar que o modelo de Belo Horizonte foi desenvolvido considerando acidentes com vítimas como variável independente, enquanto o modelo de Fortaleza e os dados de calibração e validação de Porto Alegre incluíram também os acidentes com danos materiais. Sendo assim, os valores de $C_{x}$ podem estar refletindo também essa característica que diferencia os bancos de dados usados.

A avaliação da transferibilidade de tais MPA foi realizada a partir dos parâmetros de dispersão $k$ da distribuição binomial negativa, escore $z$ e MAD e das análises dos gráficos de frequências de acidentes previstas versus observadas e de resíduos acumulados. Os resultados desta avaliação indicam que o modelo calibrado a partir da expressão de Fortaleza teve desempenho superior ao de Belo Horizonte. Esta aparente superioridade pode estar relacionada ao fato de que os coeficientes de calibração das variáveis VDMA e número de faixas serem diferentes para representar todos os acidentes ao invés de somente os acidentes com vítimas feridas e fatais como foi o caso de Belo Horizonte.

Com base nos resultados deste estudo, acredita-se que a transferência de MPA entre diferentes jurisdições é uma alternativa que deve ser usada com parcimônia, sendo desejável investir esforços no desenvolvimento de modelos específicos para cada jurisdição. Embora o $\mathrm{C}_{\mathrm{x}}$ tenha a finalidade de ajustar as diferenças de características entre as cidades/regiões para a qual o modelo foi desenvolvido e calibrado, fica-se limitado a explicar a acidentalidade com base nas variáveis adotas no modelo desenvolvido. Assim, não é possível, na transferência, testar novas variáveis explicativas que poderiam ser mais eficientes na previsão dos acidentes destas novas jurisdições.
Como sugestão para estudos futuros, recomenda-se que seja adotada como variável independente dos MPA apenas os acidentes com vítimas (feridas e fatais). Esta recomendação se dá devido à maior confiabilidade desse tipo de registro. Se por um lado os registros de acidentes com dados materiais são mais abundantes proporcionando maior quantidade de observações para a modelagem, por outro, a menor confiabilidade destes dados pode conduzir à estimação de parâmetros que não representem fidedignamente a realidade. Isto pode ocorrer, pois acredita-se que os níveis de subregistro de acidentes com danos materiais tendem a ser diferentes entre as cidades de geração e calibração dos modelos, podendo gerar distorções indesejadas nos resultados da modelagem.

Por fim, as diferenças constatadas entre as três jurisdições brasileiras ressaltam a necessidade de um estudo mais aprofundado em relação à validade dos componentes propostos na metodologia do HSM para rodovias nacionais, notadamente os modelos de previsão de acidentes e os fatores modificadores de acidentes (CMF). Acredita-se que as etapas iniciais para esse esforço de validação dependem da melhoria da qualidade global dos bancos de dados de acidentes de trânsito e da disseminação das técnicas de modelagem estatística da segurança viária na comunidade técnica brasileira, principalmente em relação ao desenvolvimento de MPA para diferentes entidades e tipos de acidentes.

\section{AGRADECIMENTOS}

Os autores agradecem o apoio do Conselho Nacional de Desenvolvimento Científico e Tecnológico ( $\mathrm{CNPq}$ ) para o desenvolvimento desta pesquisa, a Autarquia Municipal de Trânsito, Serviços Públicos e Cidadania de Fortaleza - AMC, à BHTRANS - Empresa de Transporte e Trânsito de Belo Horizonte e à Empresa Publica de Transportes e Circulação de Porto Alegre - EPTC/POA pelas informações utilizadas neste trabalho.

\section{REFERÊNCIAS}

AASHTO (2010) Highway Safety Manual. American Association of State Highway and Transportation Officials.1st. Edition, Washington, D.C. 
Barbosa, H.M., F. Cunto, B. Bezerra, C. Nodari, M.A. Jacques (2013) Perspectives on the development of safety performance models for Brazilian roads. In: Proceedings of the 13th World Congress on Transportation Research, Rio de Janeiro.

Claude, G. F. de M. (2012) Previsão da ocorrência de acidentes de trânsito em interseções de vias arteriais urbanas - $O$ caso de Taguatinga/DF. Dissertação Mestrado. Programa de Pós-Graduação em Transportes, Universidade de Brasília.

Costa, T.G., H.M. Barbosa (2011) Modelos de Previsão de Acidentes de Trânsito em Vias Urbanas de Belo Horizonte. Anais do XXV ANPET - Congresso de Pesquisa e

Ensino em Transportes, Belo Horizonte, p. 1-10.

Cunto, F.J.C., M.M. Castro Neto, D.S. Barreira (2012) Modelos de previsão de acidentes de trânsito em interseções semaforizadas de Fortaleza. Transportes, v.20, n.2, p.57-64. DOI:10.4237/transportes.v20i2.558.

Cunto, F.J.C. (2008) Assessing Safety Performance of Transportation Systems using Microscopic Simulation Tese de Doutoramento, Department of Civil and Environmental Engineering, Universidade de Waterloo, Ontário.

Hauer, E. (1997) Observational Before-After Studies in Road Safety. Pergamon Press, Elsevier Science Ltd., Oxford, England.

Holz, R.F., Medeiros, F.S., Lange, R.L., Nodari, C.T., Lindau, L.A. (2012) Volume Diário Médio Anual (VDMA): Questões Conceituais e Cálculo de Fatores de Expansão. Anais do XXV ANPET - Congresso de Pesquisa e Ensino em Transportes, Joinville, SC.
Sacchi, E., Persaud, B, Bassani, M. (2012) Assessing International Transferability of Highway Safety Manual Crash Prediction Algorithm and Its Components. Transportation Research Record: Journal of the Transportation Research Board, No. 2279, Transportation Research

Board of the National Academies, Washington, D.C., pp. 90-98. DOI: 10.3141/2279-11

Sawalha, Z. e T. Sayed (2006) Traffic accident modeling: some statistical issues. Canadian Journal of Civil Engineering.v.33, p. 1115-1124. DOI: 10.1139/I06-056

Sun, X., Li, Y., Magri, D., Shirazi, H. (2006) Application of Highway Safety Manual Draft Chapter: Louisiana Experience. Transportation Research Record: Journal of the Transportation Research Board, No. 2080, Transportation Research Board of the National Academies, Washington, D.C., p 55-64. DOI:10.3141/1950-07

Vogt, A., J. Bared (1998) Accident models for two-lane rural segments and intersections. Transportation Research Record: Journal of the Transportation Research Board, No. 1635, Transportation Research Board of the National Academies, Washington, D.C., p. 18-29. DOI: 10.3141/1635-03

Xie, F., Gladhill, K., Dixon, K. K., Monsere, C.M. (2011) Calibrating the Highway Safety Manual Predictive Models for Oregon State Highways. Transportation Research Record: Journal of the Transportation Research Board, No. 2241, Transportation Research Board of the National Academies, Washington, D.C., 2011. pp 19-28. DOI: 10.3141/2241-03 